\title{
Seroreactive species-specific lipooligosaccharides of Mycobacterium mucogenicum sp. nov. (formerly Mycobacterium chelonae-like organisms): identification and chemical characterization
}

\author{
Manuel Muñoz, ${ }^{1,2}$ Catherine Raynaud, ${ }^{2}$ Marie-Antoinette Lanéelle, ${ }^{2}$ \\ Esther Julián, ${ }^{1}$ Luz María López Marín, ${ }^{2}+$ Gaby Silve, ${ }^{2}$ Vicente Ausina, ${ }^{3}$ \\ Mamadou Daffé ${ }^{2}$ and Marina Luquin'
}

1 Departamento de Genética y de Microbiología, Unidad de Microbiología, Facultad de Ciencias, Universidad Autónoma de Barcelona, 08193 Bellaterra (Barcelona), Spain

2 Institut de Pharmacologie et de Biologie Structurale du CNRS, 205 route de Narbonne, 31077 Toulouse cedex, France

3 Servicio de Microbiología, Hospital Universitario Germans Trias i Pujol, 08916 Badalona, (Barcelona), Spain
Author for correspondence: Marina Luquin. Tel: +34 3 5812540. Fax: +34 35812387. e-mail: ibmi7@cc.uab.es

Strains of the new species Mycobacterium mucogenicum exhibit physiological and biochemical features very similar to those of the other species of the Mycobacterium fortuitum complex. To define taxonomic criteria for easy identification of $M$. mucogenicum, the glycolipid patterns of the reference strains and of 32 environmental and clinical isolates were examined by TLC. It was concluded that all $\boldsymbol{M}$. mucogenicum strains of smooth colony morphology contained species-specific alkali-labile glycoconjugates. Three different patterns were observed among the strains of the smooth colony type. Fractionation followed by conventional chemical analyses of the purified glycolipids showed the specific glycolipids to be lipooligosaccharides (LOS). The three LOS showed a similar fatty acid composition consisting of straight chain (dodeca-, tetradeca-, hexadecanoyl and hexadecenoyl) and methylbranched (2,4-dimethyleicosanoyl and 2,4-dimethyleicosenoyl) fatty acyl substituents. The most commonly encountered LOS (present in $76 \%$ of the smooth strains) contained a tetraacylated pentasaccharide composed of four moles of glucose and one mole of a 2,4-di-O-methylhexose. A LOS composed of arabinose, glucose and mannose was present in $20 \%$ of the smooth strains, whereas the newly proposed type strain of $M$. mucogenicum (ATCC 49650) was the only strain that contained a LOS composed of glucose and galactose. Serological studies clearly differentiated most of the strains of $\boldsymbol{M}$. mucogenicum from those of the other members of the $M$. fortuitum complex, and demonstrated the existence of serovars within the former species. Altogether, these data confirm the validity of the new species but show ATCC 49651 to be the most representative strain.

Keywords: Mycobacterium mucogenicum, lipooligosaccharides, inhibition ELISA, seroreactivity

\section{INTRODUCTION}

The rapidly growing non-pigmented mycobacteria are a large and complex group of environmental species,

Abbreviations: GPL, glycopeptidolipid; LOS, lipooligosaccharide; MCLO, Mycobacterium chelonae-like organisms; PIM, phosphatidylinositomannoside.

†Present address: Instituto de Investigaciones Biomédicas, Apdo Postal 70228, Universidad Nacional Autónoma de México, C.U. 04510, Mexico. some of which cause human disease. The most important pathogens among these are the members of the Mycobacterium fortuitum complex. Six taxonomic groups are recognized in this complex. These are $M$. fortuitum, Mycobacterium peregrinum, Mycobacterium chelonae, Mycobacterium abscessus and two unnamed groups, one of which is the M. fortuitum third biovariant complex, and the other the Mycobacterium chelonae-like organisms (MCLO). They are recognized as a cause of numerous nosocomial infections, including 
surgical wound infection following cardiac surgery and augmentation mammaplasty, and catheter-related infections. Pulmonary and disseminated cutaneous diseases are also caused by these mycobacteria (Wallace, 1994; Wallace et al., 1983). MCLO have been implicated in nosocomial outbreaks of peritonitis which occurred in 1976 and 1978 in Washington State, involving the use of hospital-based automated chronic peritoneal dialysis machines (Band et al., 1982). MCLO were later identified as a cause of nosocomial infections associated with haemodialysis in Louisiana (Bolan et al., 1985) and California (Lowry et al., 1990). In all cases, MCLO were recovered from tap water used in the dialysis process. MCLO are a group of mycobacteria common in potable water, more specifically tap water. A collection of 87 sporadic clinical isolates of these organisms has been recently characterized (Wallace et al., 1993). The majority of these isolates $(62 \%)$ were respiratory isolates, and two strains were isolated from patients with AIDS. Among 33 non-respiratory isolates, 20 were of clinical significance.

Based on their morphological and biochemical characteristics, MCLO were included in the $M$. fortuitum complex (Lévy-Frébault et al., 1983; Silcox et al., 1981; Wallace et al., 1993). More recently, however, the name Mycobacterium mucogenicum sp. nov. was proposed for this group (Springer et al., 1995) on the basis of their rRNA gene sequences. This proposal is in agreement with the mycolic acid pattern of MCLO, which has been shown to be unique within the $M$. fortuitum complex (Lévy-Frébault et al., 1983; Muñoz et al., 1997; Wallace et al., 1993), but further studies are still needed to clarify the taxonomic status of $M$. mucogenicum.

Earlier studies have demonstrated that most mycobacterial species contain large quantities of a variety of complex lipids, including species-specific glycoconjugates, which possess a potential value in taxonomy, epidemiology and serodiagnosis, and may also play a role in the pathogenicity of these organisms (for a review see Brennan et al., 1990). In recent years examination of the glycolipid content of members of the $M$. fortuitum complex has greatly contributed to the knowledge of this closely related group of organisms, both in terms of taxonomic relatedness and of structures of compounds elaborated by the members of the group. For instance, a class of antigenic mycobacterial glycolipids belonging to the family of C-mycoside glycopeptidolipids (GPLs) has been identified in $M$. abscessus, $M$. chelonae, $M$. peregrinum and some strains of the $M$. fortuitum third biovariant complex (Lanéelle et al., 1996; López Marín et al., 1991, 1994; Tsang et al., 1984). Chemical analysis, seroagglutination and ELISA have established that the GPLs of $M$. chelonae and $M$ abscessus have identical structures and differ from those of $M$. peregrinum and the M. fortuitum third biovariant (Lanéelle et al., 1996; López Marín et al., 1991, 1994; Tsang et al., 1984). In contrast, a lipooligosaccharide (LOS) has been characterized in some strains of the third biovariant complex of M. fortuitum (Lanéelle et al., 1996), whereas two different specific antigenic acylated trehaloses, a diacyl- and a triacyltrehalose, have been described in $M$. fortuitum (Ariza et al., 1994; Gautier et al., 1992; Sempere et al., 1993). Thus, the analysis of the glycolipid content of members of the $M$. fortuitum complex validated the DNA-DNA homology studies which had recently established that $M$. fortuitum and $M$. peregrinum belonged to two different species (Kusunoki \& Ezaki, 1992; Lévy-Frébault et al., 1986). Previously they had been considered as biovarieties of the same species (M. fortuitum bv. fortuitum and M. fortuitum bv. peregrinum, respectively). The glycolipid analysis also confirmed the heterogeneity of the strains grouped in the so-called $M$. fortuitum third biovariant complex, which have been shown to differ one from the other by biochemical tests, genetic studies and sensitivity to antimicrobial drugs (Kirschner et al., 1992; Wallace et al., 1991). Consequently, we investigated the glycolipid content of three reference strains and 32 clinical and environmental isolates of $M$. mucogenicum, the most recent species to be included in the $M$. fortuitum complex, in order to: (i) evaluate the homogeneity of the lipid pattern of strains belonging to this species; (ii) evaluate the antigenicity of the crude lipids of $M$. mucogenicum and define the chemical basis of the seroreactivities; (iii) define the taxonomic relatedness of the species to the other members of the M. fortuitum complex on the basis of the class of species-specific glycolipids synthesized by $M$. mucogenicum and of the serological cross-reactions.

\section{METHODS}

Bacterial strains, media and growth conditions. This study was performed with three reference strains of $M$. mucogenicum (ATCC 49649, ATCC 49650 and ATCC 49651) and 32 strains isolated from clinical and environmental sources (Table 1). These isolates were identified as M. mucogenicum by standard cultural, biochemical and physiological procedures as described previously (Muñoz et al., 1997). Their mycolic acid patterns, and fatty acid and alcohol profiles were determined by TLC and GC, respectively. In addition, the characterization of the hypervariable region within the $16 \mathrm{~S}$ rRNA (Muñoz et al., 1997) was performed in selected isolates.

All strains were grown on plates of Sauton's agar (Sauton, 1912) incubated at $33^{\circ} \mathrm{C}$. Isolated colonies of 2 -week-old cultures were examined for colony characteristics with a stereomicroscope and an external light source. For the isolation of glycolipids, cells from 2-week-old cultures of either pure rough or smooth colonies of M. mucogenicum were harvested from plates by scraping. For comparison, the type strains of $M$. chelonae (NCTC 946), $M$. fortuitum (ATCC 6841), M. peregrinum (ATCC 14467) and a reference strain of the $M$. fortuitum third biovariant complex (ATCC 49403) were grown on Sauton's medium as surface pellicles in $250 \mathrm{ml}$ glass flasks at $33^{\circ} \mathrm{C}$ for 2 weeks, and the cells were harvested as previously described (Lanéelle et al., 1996; López Marín et al., 1994).

Extraction and purification of glycolipids. Glycolipids were extracted from harvested bacteria (approximately $2 \mathrm{~g}$ wet $\mathrm{wt}$ cells) as described by Daffé et al. (1991). The glycolipid content of lipid extracts thus obtained was examined by TLC 
on silica-gel-coated plates (G-60, $0.25 \mathrm{~mm}$ thickness, Merck) developed with chloroform/methanol $(90: 10, \mathrm{v} / \mathrm{v})$ or with chloroform/methanol/water $(30: 8: 1,65: 25: 4$ or $60: 35: 8$, by vol.). The glycolipids were visualized by spraying plates with $0 \cdot 2 \%(\mathrm{w} / \mathrm{v})$ anthrone in concentrated $\mathrm{H}_{2} \mathrm{SO}_{4}$, followed by heating at $120^{\circ} \mathrm{C}$. Molybdenum blue spray reagent (Sigma) was used for revealing phosphorus-containing substances. The lipid extracts were dissolved in chloroform and applied to a column of silica gel (70-230 mesh, Merck) equilibrated in chloroform. The column was first eluted with chloroform, and then with increasing concentrations of methanol in chloroform $(5,10,20,30,50$ and $100 \%, v / v)$. The different eluates were concentrated and examined by TLC using the eluting solvents mentioned above. The chromatographic fractions eluted with chloroform/methanol $(1: 1, \mathrm{v} / \mathrm{v})$ and $100 \%$ methanol contained the most polar glycolipids, consisting of phospholipids and some anthrone-reactive substances. These two fractions were pooled and applied on a DEAE-cellulose column (Merck) to purify the unidentified glycoconjugates. The DEAE-cellulose column was eluted with chloroform and with chloroform containing increasing concentrations of methanol, and the purification process was monitored by TLC.

\section{Immunological procedures}

Preparation of antiserum. Rabbit antisera were obtained by intradermal injection (in multiple sites) of $1 \mathrm{ml}$ of a water-inoil emulsion prepared by mixing $0.5 \mathrm{ml}$ PBS $(0.145 \mathrm{M} \mathrm{NaCl}$, $0.01 \mathrm{M} \mathrm{K} \mathrm{K}_{2} \mathrm{HPO}_{4} / \mathrm{KH}_{2} \mathrm{PO}_{4}, \mathrm{pH} 7 \cdot 2$ ) containing $5 \mathrm{mg}$ lipid extract of either strain 51 or $54 \mathrm{~s}$ of $\mathrm{M}$. mucogenicum and $0.5 \mathrm{ml}$ Freund's incomplete adjuvant. The emulsion was injected into 3-month-old New Zealand White rabbits. Three weeks later, the animals received a booster dose of $3 \mathrm{mg}$ lipid extract in water-in-oil emulsion, as described above, and were then bled at 2-week intervals.

Direct ELISA. Lipids were suspended at $500 \mu \mathrm{g} \mathrm{ml}^{-1}$ in hexane/ ethanol $(1: 1, \mathrm{v} / \mathrm{v})$. Samples $(50 \mu \mathrm{l})$ were applied to the wells of a polystyrene microtitre plate (Nunc-Immuno Plate I) and allowed to evaporate at $37^{\circ} \mathrm{C}$ overnight. After saturation and washing, $100 \mu \mathrm{l}$ of the rabbit sera, diluted $1: 1000(\mathrm{v} / \mathrm{v})$ in $0.4 \%(\mathrm{w} / \mathrm{v})$ powdered skimmed milk in PBS, was added and the plates incubated for $2 \mathrm{~h}$ at $37^{\circ} \mathrm{C}$. After washing, alkaline phosphatase conjugated to whole anti-rabbit IgG (Sigma) and $p$-nitrophenyl phosphate substrate (Sigma) were used for the colour reaction. Absorbance was measured at $410 \mathrm{~nm}$ with a Multiskan apparatus (Flow Laboratories). Each value was measured in triplicate.

Inhibition ELISA. The inhibition of the reaction between $25 \mu \mathrm{g}$ solid-phase lipid extract of either strain 51 or 54 s of $M$. mucogenicum and specific rabbit anti-(lipids of the given strain of M. mucogenicum) serum diluted 1:100 (v/v) in PBS by the whole lipid extract, the purified alkali-labile glycolipid isolated from strain 51 or by D-glucose, D-mannose, D- or Larabinose was performed as follows. The inhibitory compounds were suspended at $500,250,125$ and $100 \mu \mathrm{g} \mathrm{ml}^{-1}$ in either hexane/ethanol $(1: 1, \mathrm{v} / \mathrm{v})$ or $0.4 \%(\mathrm{w} / \mathrm{v})$ powdered skimmed milk in PBS. Then, $50 \mu$ l of these suspensions, $40 \mu \mathrm{l}$ of $0.4 \%(\mathrm{w} / \mathrm{v})$ powdered skimmed milk in PBS and $10 \mu \mathrm{l}$ rabbit anti-(lipids of either strain 51 or $54 \mathrm{~s}$ of $\mathrm{M}$. mucogenicum) serum (diluted 1:100, v/v, in PBS) were incubated for $2 \mathrm{~h}$ at $37^{\circ} \mathrm{C}$; the mixtures were deposited in wells that had previously been coated with $25 \mu \mathrm{g}$ whole lipid extract from the given strain and blocked by $200 \mu \mathrm{l} 30 \%$ (w/v) powdered skimmed milk in PBS. The mixture was left for $2 \mathrm{~h}$ at $37^{\circ} \mathrm{C}$ before rinsing. The colour reaction used anti-rabbit IgG (Sigma) and $p$-nitrophenyl phosphate substrate (Sigma) as above. A control for no inhibition was performed using $10 \mu \mathrm{l}$ serum diluted $1: 100(\mathrm{v} / \mathrm{v})$ in PBS and $90 \mu \mathrm{l}$ buffer; a blank control was obtained by the development of the complete ELISA reaction in wells not coated with lipids.

\section{Analytical techniques}

Alkaline deacylation. To determine the stability of the compounds towards alkali, the crude lipid extracts and the purified glycolipids were deacylated according to Brennan \& Goren (1979).

Sugar characterization. Samples (1-5 mg) were hydrolysed with $2 \mathrm{M} \mathrm{CF}_{3} \mathrm{COOH}$ solution at $110^{\circ} \mathrm{C}$ for $4 \mathrm{~h}$. After evaporation, the hydrolysates were partitioned between diethyl ether and water. The aqueous phase was recovered and divided into two portions. One portion was dried under nitrogen and trimethylsilylated (Sweeley et al., 1963). The remaining portion of the aqueous phase was reduced by $\mathrm{NaBH}_{4}$, and peracetylated with acetic anhydride/pyridine $(1: 1, \mathrm{v} / \mathrm{v})$ overnight at room temperature. The trimethysilylated and alditol acetate derivatives of sugars were analysed by GC.

Fatty acid composition of glycolipids. The organic phases from the acid hydrolysis and from the alkaline deacylation (as described above) were treated with diazomethane. The resulting fatty acid methyl esters were analysed by GC and GC-MS.

GC and GC-MS. GC and GC-MS analyses of fatty acid methyl esters and both alditol acetate and trimethylsilyl derivatives of saccharides were performed on a Hewlett-Packard 5890A chromatograph connected to a Hewlett-Packard 5989X mass selective detector using a fused-silica capillary column (12 $\mathrm{m} \times 0.25 \mathrm{~mm}$ internal diameter) with cross-linked methyl silicone (HP-1, Hewlett-Packard) as the stationary phase. The injector and transfer line temperatures were 275 and $280^{\circ} \mathrm{C}$ respectively. The carrier gas was helium with a flow rate of $1 \mathrm{ml} \mathrm{min}$, and the split ratio was approximately 1:50. For the analysis of fatty acid methyl esters, the oven was

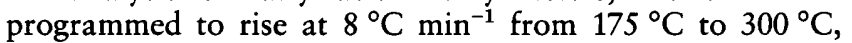
followed by a $15 \mathrm{~min}$ hold at $300^{\circ} \mathrm{C}$. The temperature programme that was applied to trimethylsilyl and alditol acetate derivatives involved a $100^{\circ} \mathrm{C}$ hold for $2 \mathrm{~min}$, followed by a $5{ }^{\circ} \mathrm{C} \mathrm{min}^{-1}$ rise to $280^{\circ} \mathrm{C}$. The chromatograms were integrated by using a HP3396 Series II Integrator (HewlettPackard). Fatty acid methyl esters and trimethylsilylated derivatives of sugars were identified by comparing their retention times with those of authentic methyl esters and alcohol standards (Supelco and Sigma). The identities of fatty acid methyl esters were also confirmed by MS.

NMR. All the NMR spectra were obtained in $\mathrm{CDCl}_{3} / \mathrm{CD}_{3} \mathrm{OD}$ $(2: 1, \mathrm{v} / \mathrm{v})$ at $25^{\circ} \mathrm{C}$. They were obtained with $40^{\circ}$ pulses, and acquisition times of $1.56 \mathrm{~s}$ for the ${ }^{13} \mathrm{C}-\mathrm{NMR}$ spectrum (spectral width $5263 \mathrm{~Hz}$ ) and $2.73 \mathrm{~s}$ for the ${ }^{1} \mathrm{H}-\mathrm{NMR}$ spectrum (spectral width $2994 \mathrm{~Hz}$ ) were used. The ${ }^{1} \mathrm{H}-\mathrm{NMR}$ spectrum was recorded on a Bruker WM 250 apparatus instrument at $250 \mathrm{MHz}$, and the ${ }^{13} \mathrm{C}-\mathrm{NMR}$ spectrum was obtained with the same instrument operating at $62.9 \mathrm{MHz}$ using a Bruker J-Mod sequence. The chemical-shift reference used was that of tetramethylsilane.

\section{RESULTS}

\section{Morphology of the colonies of $\boldsymbol{M}$. mucogenicum}

One physical characteristic of M. mucogenicum, also shown by other mycobacteria, is an ability to exhibit various colony morphologies (rough and variable smooth formats). The three reference strains, as well as 
Table 1. Origin, colony morphology and LOS pattern of $M$. mucogenicum reference strains and isolates

\begin{tabular}{|c|c|c|c|}
\hline Strain no. & Source & $\begin{array}{c}\text { Colonial } \\
\text { morphology }\end{array}$ & LOS pattern $\nmid$ \\
\hline ATCC 49649 & Peritoneal fluid & Smooth & I \\
\hline ATCC 49650 & Neck abscess & Smooth & III \\
\hline ATCC 49651 & $\begin{array}{l}\text { Postinjection buttock } \\
\text { abscess }\end{array}$ & Smooth & I \\
\hline 51 & Gastric lavage & Smooth & I \\
\hline $54 \mathrm{~s}$ & Urine & Smooth & II \\
\hline $54 \mathrm{r}$ & Urine & Rough & - \\
\hline 147 & Gastric lavage & Smooth & II \\
\hline 1 & River mouth & Smooth & $\mathrm{I}$ \\
\hline $2 s$ & River mouth & Smooth & I \\
\hline $2 r$ & River mouth & Rough & - \\
\hline $32 s$ & River mouth & Smooth & $\mathrm{I}$ \\
\hline $32 \mathrm{r}$ & River mouth & Rough & - \\
\hline 33 & River mouth & Smooth & II \\
\hline 3 & Seawater & Smooth & I \\
\hline 4 & Seawater & Smooth & II \\
\hline 5 & Seawater & Smooth & I \\
\hline 11 & Seawater & Smooth & I \\
\hline 12 & Seawater & Smooth & I \\
\hline 13 & Seawater & Smooth & I \\
\hline 14 & Seawater & Smooth & I \\
\hline 15 & Seawater & Smooth & I \\
\hline 20 & Seawater & Smooth & I \\
\hline 34 & Seawater & Smooth & I \\
\hline 35 & Seawater & Smooth & I \\
\hline 45 & Seawater & Rough & - \\
\hline 46 & Seawater & Smooth & I \\
\hline 98 & Sewage water & Rough & - \\
\hline 102 & Sewage water & Smooth & I \\
\hline $106 \mathrm{~s}$ & Sewage water & Smooth & II \\
\hline $106 \mathrm{r}$ & Sewage water & Rough & - \\
\hline 112 & Sewage water & Rough & - \\
\hline 131 & Sewage water & Rough & - \\
\hline 205 & Sewage water & Rough & - \\
\hline 207 & Sewage water & Rough & - \\
\hline 208 & Sewage water & Smooth & I \\
\hline
\end{tabular}

* River mouth, at the mouth of the River Besos, one of the two rivers that flow through the suburbs of Barcelona; seawater, at different points in coastal waters about $200 \mathrm{~m}$ offshore; sewage water, at the admittance and discharge points of the sewage treatment plant at the town of Vilafranca del Penedès, situated about $50 \mathrm{~km}$ from Barcelona.

† Patterns I-III defined by the sugar composition of the LOS-containing fractions; - indicates no pattern detected.

22 out of the 32 strains that we isolated, displayed a smooth morphology (Table 1, Fig. 1a, b, c). The remaining 10 strains showed a rough morphology (Table 1, Fig. 1d). Three types of smooth colonies were observed (Fig. 1). The reference strains ATCC 49649 and ATCC 49651, and all the smooth isolates that elaborated the glycolipid pattern I (Table 1), formed circular, convex colonies with entire margins (Fig. 1a), whereas the strains which contained glycolipids of pattern II (Table 1) exhibited irregular, raised colonies with complex margins (Fig. 1b). The newly proposed type strain ATCC 49650 was the only strain examined that formed circular, convex colonies with a slightly translucent border (Fig. 1c) and showed the glycolipid pattern III (Table 1).

Among the strains isolated from human clinical specimens, the pure cultures (51 and 147) exhibited a smooth morphology; the remaining two strains $(54 \mathrm{~s}, 54 \mathrm{r})$ were isolated from an originally mixed culture in which the 

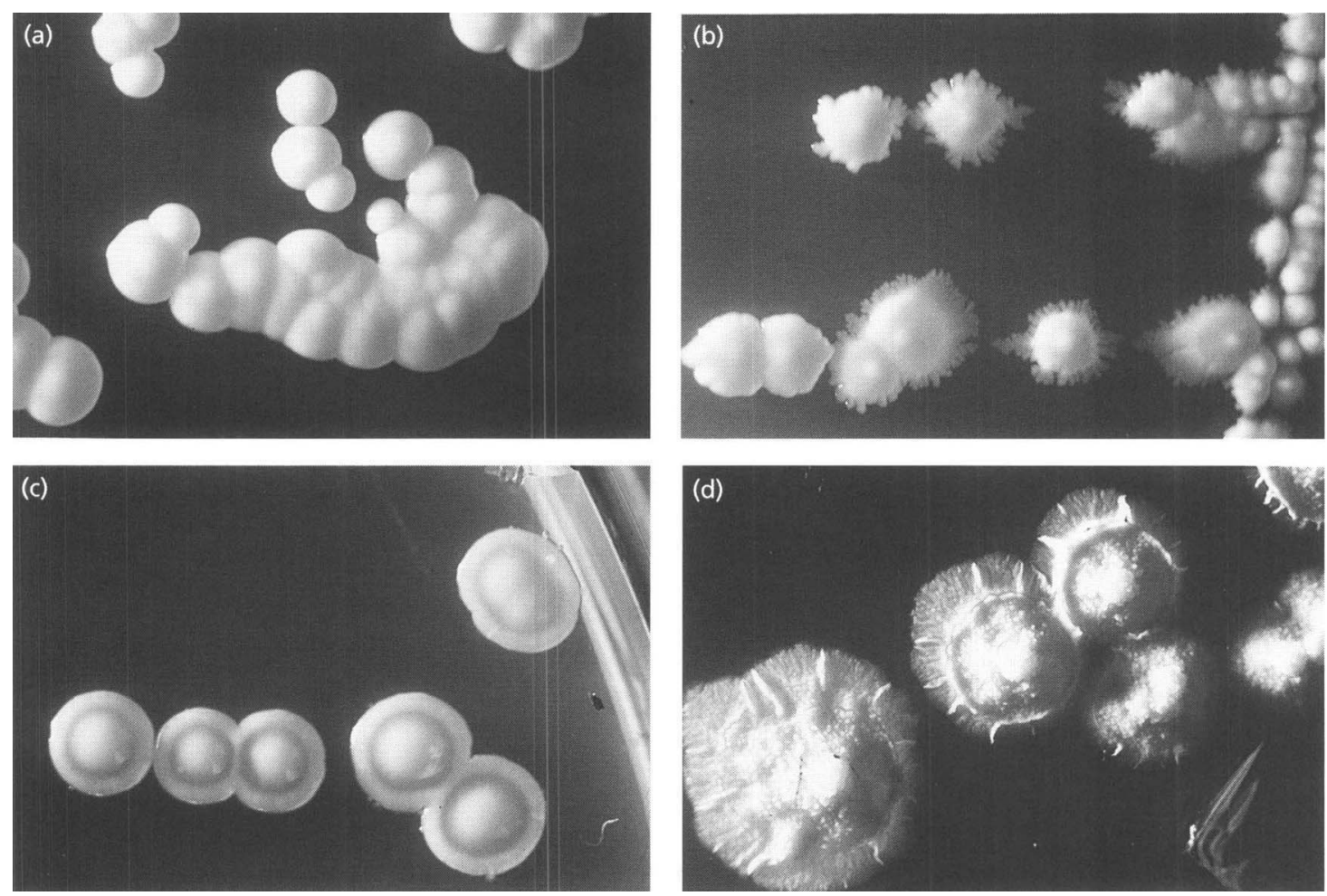

Fig. 1. Smooth colonies of strain ATCC 49649 and related isolates (a), isolate 54s (b) and strain ATCC 49650 (c), and rough colonies of $M$. mucogenicum (d).

strain with smooth morphology (54s) was predominant. The isolation of mixed cultures in which the smooth strain predominated over the rough strain also occurred in two aquatic isolates, i.e. the pairs $2 \mathrm{~s} / 2 \mathrm{r}$ and $32 \mathrm{~s} / 32 \mathrm{r}$. Interestingly, this predominance of the strains with smooth morphology over the strains with rough morphology did not occur in the strains isolated from sewage water, a highly contaminated and perhaps more hostile environment. Of the nine strains isolated from sewage water, six displayed a rough morphology and three exhibited a smooth morphology. In the only mixed culture obtained from sewage water (strains 106s and $106 r$ ), the strain with a rough morphology predominated over the strain with a smooth one. The predominance of a given morphology in mixed cultures according to the origin of isolations (water-based environment, clinical specimens or sewage water) suggest that, in the case of M. mucogenicum, external factors may influence the production of smooth or rough colonies.

\section{Identification of LOS in smooth strains of M. mucogenicum}

Comparative TLC analyses of the lipid extracts of the various strains showed, as expected, the presence of ubiquitous mycobacterial lipids in all the strains examined. These included triacylglycerol, mycolate-containing glycolipids and phospholipids. In addition, one or more major spots which correspond to polar lipids, exhibiting chromatographic mobilities similar to those of phosphatidylinositomannosides (PIMs) were detected from all the smooth strains of M. mucogenicum (Fig. 2); all the rough strains were devoid of these spots. The additional spots readily reacted with anthrone, a reagent that specifically reveals the presence of carbohydrates, but not with molybdenum blue, a specific reagent for phosphorylated substances (Fig. 2). Furthermore, the additional spots disappeared after treatment of lipids from smooth strains of $M$. mucogenicum with $0.2 \mathrm{M}$ $\mathrm{NaOH}$ in methanol (a reaction that cleaves ester links). Thus, these preliminary data demonstrated that the substances present in smooth strains, and absent in the rough strains of M. mucogenicum (Fig. 2), conformed to the specification of acylated oligosaccharides, more commonly known as LOS (McNeil et al., 1989).

Three different LOS patterns were clearly observed by TLC (Fig. 2, Table 1). Pattern I was shared by most $(76 \%)$ of the smooth strains examined, including the two reference strains ATCC 49649 and ATCC 49651 (Fig. 2, lanes 1, 3 and 4-7). All these strains formed circular, convex colonies with entire margins (Fig. 1a). Pattern II (Fig. 2, lane 8) was observed in the five strains $(20 \%$ of the smooth strains) which formed irregular, raised colonies with complex margins (Fig. 1b), whereas the third pattern (Fig. 2, lane 2) was exclusively found in the type strain (ATCC 49650), which formed circular and convex colonies with a slightly translucent border (Fig. 1c). These patterns were found to have been invariant during the last 2 years; no change has ever 


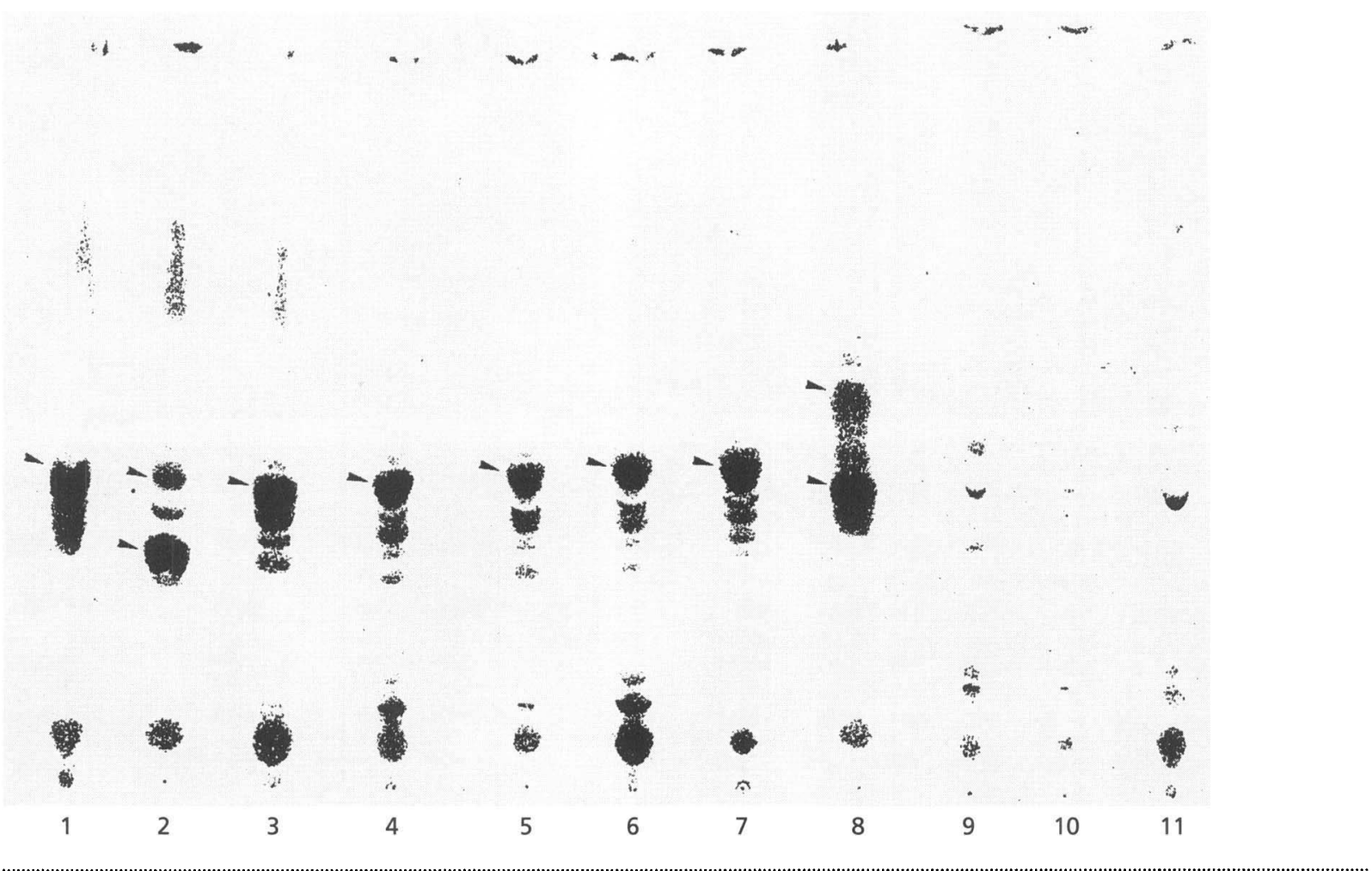

Fig. 2. TLC (solvent, chloroform/methanol/water, 60:35:8, by vol.; colour reagent, anthrone) of the lipid extracts of the reference strains ATCC 49649 (lane 1), ATCC 49650 (lane 2), ATCC 49651 (lane 3) of M. mucogenicum and of smooth strains 2s, 32s, 51, 102 and 54s (lanes 4-8, respectively) and rough isolates 54r, 2r and 32r (lanes 9-11, respectively). Arrows indicate phosphorus-free alkali-labile glycolipids (LOS). The compounds exhibiting chromatographic mobilities similar to that of LOS correspond to PIMs.

been detected in the LOS pattern of the various strains studied.

\section{Purification and chemical composition of the major LOS of $M$. mucogenicum}

The lipid extracts of representative strains of LOS pattern I (strain 51 and ATCC 49649), pattern II (strain 54s) and pattern III (strain ATCC 49650), as well as those of some rough strains ( $2 r$ and $32 r$ ) were fractionated by adsorption chromatography on silica gel columns to yield enriched LOS fractions. The neutral polar glycolipids (LOS) were separated from the PIMs by anion-exchange chromatography on DEAE-cellulose columns. Application of this procedure yielded pure LOS from strains exhibiting LOS patterns I and II. In the case of strain ATCC 49650 (LOS pattern III), however, LOS were eluted with PIMs; application of various chromatograhic methods failed to give pure LOS, suggesting the occurrence of acidic substituents.

Analysis of the fatty acid methyl esters that result from the alkaline deacylation of LOS by GC and GC-MS showed a similar profile for the three compounds (Fig. $3)$. The fatty acyl substituents were identified on the basis of their retention times on GC, the $m / z$ values of their molecular ion peaks, $[\mathrm{M}]^{+}$, and of the fragment ion peaks, $\left[\mathrm{M} \text { minus } \mathrm{C}_{2}-\mathrm{C}_{3}-\mathrm{C}_{4}\right]^{+}$, on GC-MS, and the occurrence of ion peaks at $m / z 74$ (or 88 ) and 87 (or 101) (Ryhage \& Stenhagen, 1960). The major fatty acyl component of the LOS was octadecenoyl $\left(\mathrm{C}_{18: 1}\right)$. Significant amounts of lower homologues $\left(C_{16}, C_{14}\right.$ and $\mathrm{C}_{12}$ ) were also present. In addition, methyl-branched fatty acyl substituents were identified. They consisted of 2-methyloctadecanoyl, 2,4-dimethyleicosenoyl and 2,4dimethyleicosanoyl. The relative amounts of the different fatty acyl substituents varied slightly according to the origin of the LOS and, presumably, the growth phase of the examined strains.

Acid hydrolysis of the different LOS followed by the analysis of the sugar derivatives by GC demonstrated that the difference in the chromatographic mobility of the various LOS (Fig. 2) corresponded to a difference in the sugar composition (Table 2), rather than to the number of fatty acyls substituting the LOS. Glucose was the major sugar constituent of the three glycolipids, as commonly found in mycobacterial LOS. The additional sugar that typified the LOS present in most of the smooth strains of M. mucogenicum, i.e. LOS pattern I, corresponded to a 2,4-di-O-methylhexose (fragment ion peaks at $m / \mathrm{z} 43,117,189,201,233$ and 305). Mannose and arabinose, in addition to glucose, were the sugar constituents of LOS pattern II (Table 2). Acid hydrolysis of the enriched LOS fraction from the type strain ATCC 49650 released glucose and galactose. In addition, 


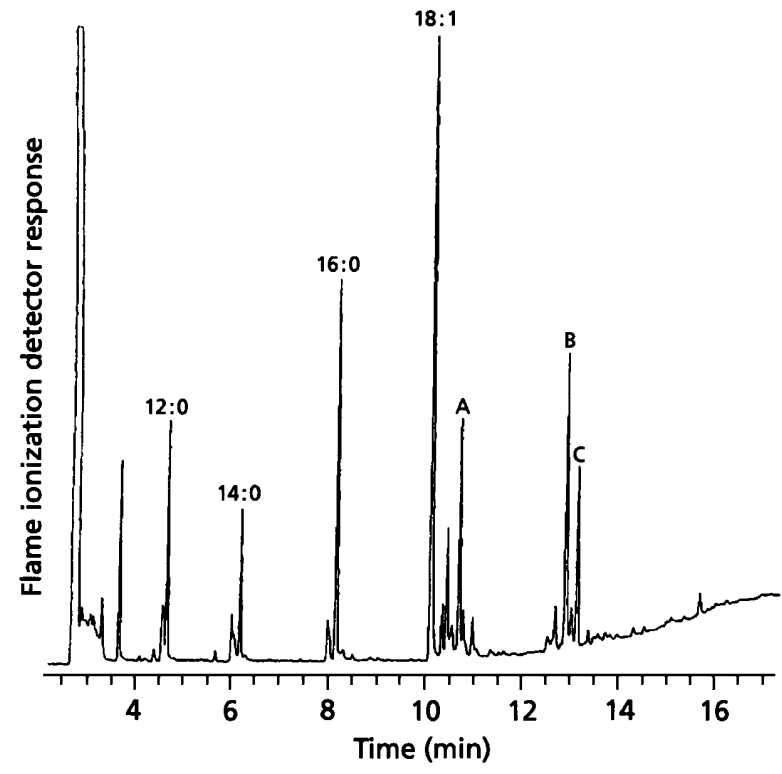

Fig. 3. GC profiles of methyl esters resulting from the deacylation of the LOS purified from isolate 51 of $M$. mucogenicum. Peaks $A, B$ and $C$ correspond to 2methyloctadecanoate, 2,4-dimethyleicosenoate and 2,4dimethyleicosanoate, respectively.

Table 2. Glycosyl composition of the different LOS patterns found in $M$. mucogenicum

+ and - indicate the presence and absence of the sugar, respectively.

\begin{tabular}{|lccc|}
\hline & Pattern I & Pattern II & Pattern III \\
\hline Glucose & + & + & + \\
Mannose & - & + & + \\
Arabinose & - & + & - \\
Galactose & - & - & + \\
2,4-Di-O- & + & - & - \\
methylhexose & & & \\
Glycerol & - & - & + \\
Inositol & - & - & + \\
\hline
\end{tabular}

glycerol, mannose and inositol, presumably derived from the contaminant PIMs (see above), were found (Table 2). Interestingly, the polyol constituents of PIMs, i.e. mannose, glycerol and inositol, were the only hydrosoluble products detected by the GC analysis of the acid hydrolysis products of the polar lipid fractions from the rough strains examined.

\section{NMR analyses of the commonest LOS}

Because LOS pattern I was encountered in the majority of the smooth strains of $M$. mucogenicum examined, further structural analyses were conducted on the purified LOS by ${ }^{1} \mathrm{H}$ - and ${ }^{13} \mathrm{C}-\mathrm{NMR}$. The ${ }^{1} \mathrm{H}-\mathrm{NMR}$ spectrum (Fig. 4a) was informative, notably in the region of anomeric and other deshielded protons
(4.5-5.5 p.p.m.). Two doublets $\left(J_{1,2}=3.5 \mathrm{~Hz}\right)$ were observed at 5.23 and 5.32 p.p.m. and assigned to the resonances of $\mathrm{H}-1$ of $\alpha$-hexopyranosyl residues possessing a gluco configuration (Daffé et al., 1988). The triplet centred at 5.07 p.p.m. may be attributed to either a deshielded $\mathrm{H}-3$ or $\mathrm{H}-4$ resonance of a glucopyranosyl unit that had an esterified hydroxyl group located on C3 or C-4. Similarly, a doublet of doublets was observed at 4.85 p.p.m., assignable to the resonance of $\mathrm{H}-2$ of an $\alpha$-glucopyranosyl residue that had an esterified hydroxyl group located on C-2 (Daffé et al., 1988, 1991; Gautier et al., 1992; Lanéelle et al., 1996). Analysis of the ${ }^{1} \mathrm{H}-\mathrm{NMR}$ spectrum of the LOS confirmed the presence of double bonds (resonance of the ethylenic protons at $5 \cdot 40$ p.p.m.), long-chain fatty acyl residues (a massive signal corresponding to the resonance of methylenic protons centred at 1.30 p.p.m.), methyl-branched protons (doublet at 1.20 p.p.m.) and other features (see Fig. 4a) that were in agreement with the data obtained by GC and GC-MS of fatty acid methyl esters (see above). In addition, two sharp singlets were observed at 3.60 and 3.70 p.p.m. and assigned to the resonances of the methyl protons of the two methoxyl groups of the 2,4-di-Omethylhexosyl residue of the LOS. Analysis of the ${ }^{13} \mathrm{C}-$ NMR spectrum (Fig. 4b) confirmed the data obtained from the interpretation of the ${ }^{1} \mathrm{H}-\mathrm{NMR}$ spectrum and demonstrated the occurrence of five glycosyl units and four acyl substituents in the LOS. Two signals, assignable only to the resonances of the two anomeric carbons of $\alpha, \alpha^{\prime}$-trehalose (Bradbury \& Jenkins, 1984), were observed at 92.60 and 94.60 p.p.m. (Fig. 4b, inset). The three other anomeric signals at 102.17, 104.40 and 104.9 p.p.m. were tentatively attributed to the di-Omethylhexopyranosyl residue and to two $\beta$-glucopyranoyl units (Bradbury \& Jenkins, 1984). The existence of four deshielded signals (at $>170$ p.p.m.), assignable to the resonances of carbonyl, indicated the presence of four acyl substituents in the glycolipid. Thus, the LOS pattern I corresponded to a tetraacylated pentasaccharide whose complete structure is under study.

\section{Seroreactivity of the lipid extracts}

Rabbit sera were raised against the whole lipid extracts from the representative strains of LOS patterns I and II (isolates 51 and $54 \mathrm{~s}$, respectively) to evaluate the antigenicity of the two LOS. In both cases the highest seroreactivities were obtained with sera produced 7 weeks after immunization. As shown in Fig. 5, the lipids from a given strain reacted readily in ELISA with the homologous serum. In contrast, the sera raised against the lipid extracts of isolates 51 and $54 \mathrm{~s}$ reacted only weakly with the heterologous lipids (Fig. 5), demonstrating the specificity of the seroreactions and confirming the existence of at least two serovars among the strains of $M$. mucogenicum. Furthermore, the sera directed against the lipids from strains 51 and 54 s, which represented $96 \%$ of the smooth strains of $\mathrm{M}$. mucogenicum, did not recognize the lipid extracts from other members of the $M$. fortuitum complex tested, which 


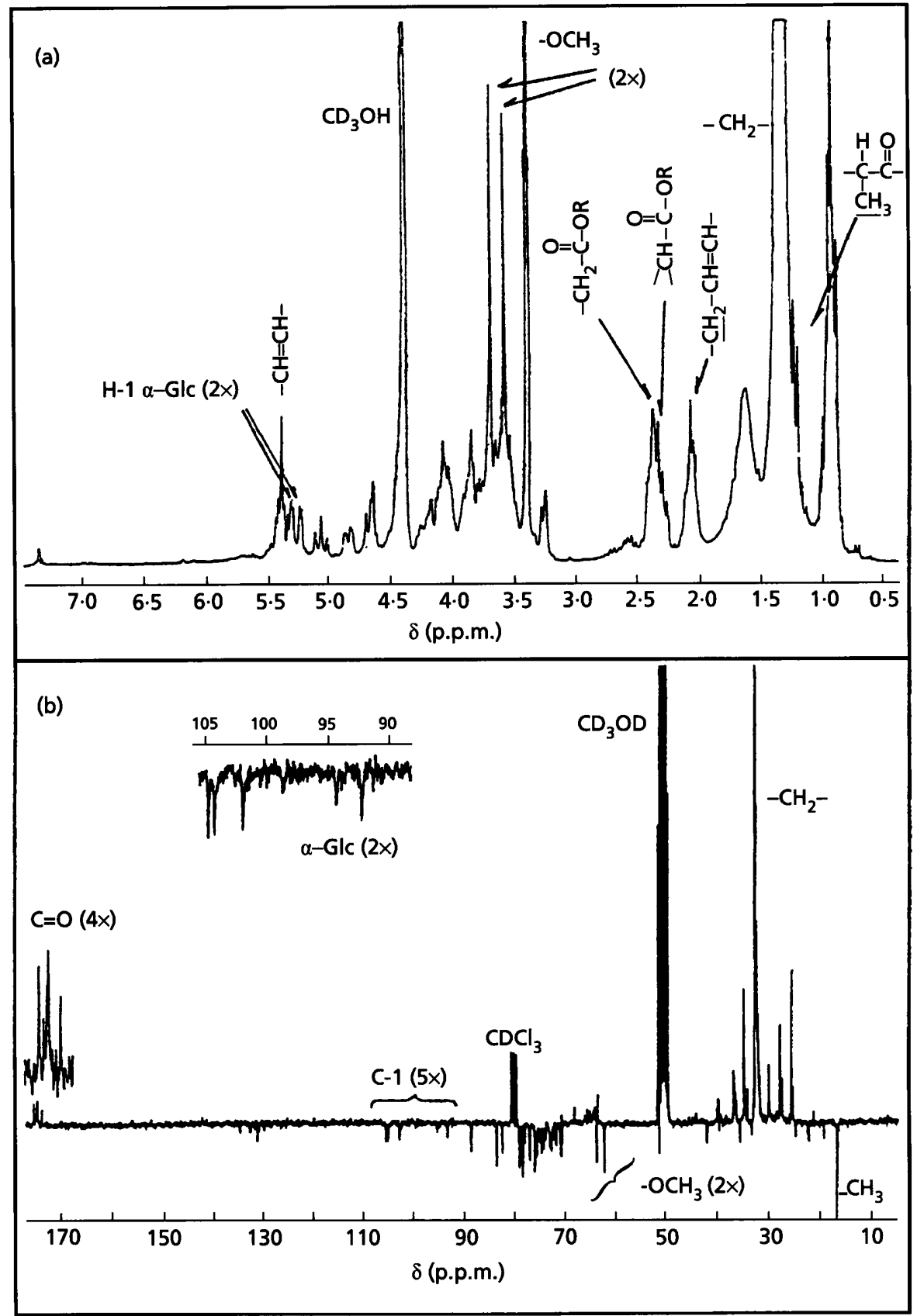

Fig. 4. NMR spectra of the LOS purified from isolate 51 of $M$. mucogenicum. (a) ${ }^{1} \mathrm{H}$-NMR spectrum; (b) portion of the $J$-modulated ${ }^{13} \mathrm{C}$-NMR spectrum. Both spectra were recorded in $\mathrm{CDCl}_{3} / \mathrm{CD} 3 \mathrm{OD}(2: 1, \mathrm{v} / \mathrm{v})$.

included the type strains of $M$. fortuitum, $M$. peregrinum and $M$. chelonae, and a representative strain of the third biovariant complex of M. fortuitum.

The seroreactivity of the lipids from isolates 51 and $54 \mathrm{~s}$ with the homologous serum was abolished by deacylation, showing that the alkali-labile substances represented the main antigenic compounds. In addition, the lipids from the different rough strains, whose composition differed from those of strains 51 and 54 s only by the absence of LOS, did not react with any of the sera. This demonstrates that the ubiquitous lipids (PIMs and mycoloyltrehaloses) did not significantly contribute to the observed seroreactivity of lipids from smooth strains, and suggests that LOS were the antigenic substances. To test this hypothesis, the contribution of the purified LOS from strain 51 to the seroreactivity was determined.

\section{Seroreactivity of the purified LOS of strain $\mathbf{5 1}$}

As expected from the seroreactivity of the various lipid extracts, the purified LOS of isolate 51 reacted strongly with the serum raised against the whole lipids from 


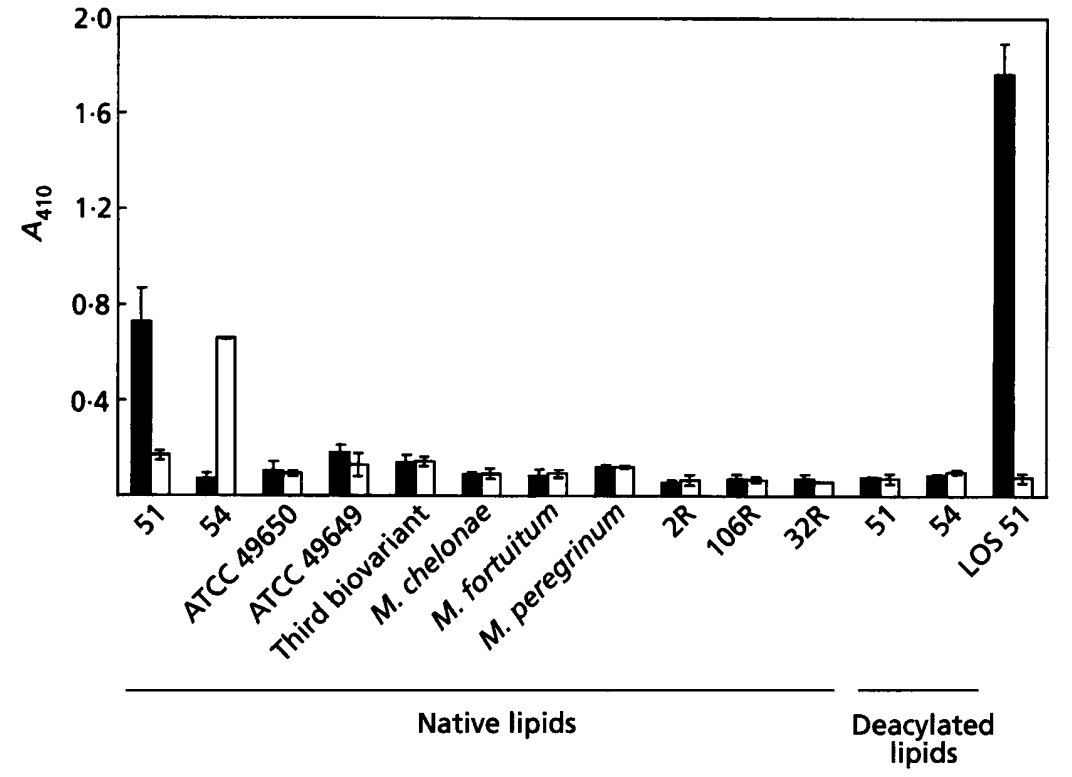

Fig. 5. Reactivity in ELISA of native lipid extracts of reference strains and isolates of $M$. mucogenicum and representative strains of the other species of the $M$. fortuitum complex, of deacylated lipids of isolates 51 and $54 \mathrm{~s}$ of $M$. mucogenicum, and of purified LOS from strain 51 against a rabbit anti(whole lipid antigens of strain 51 ) serum (black columns) and a rabbit anti-(whole lipid antigens of strain 54s) serum (white columns). Results represent the arithmetic mean for three determinations and $25 \mu \mathrm{g}$ product was used per well. The sera were diluted in PBS (1:1000). (a)

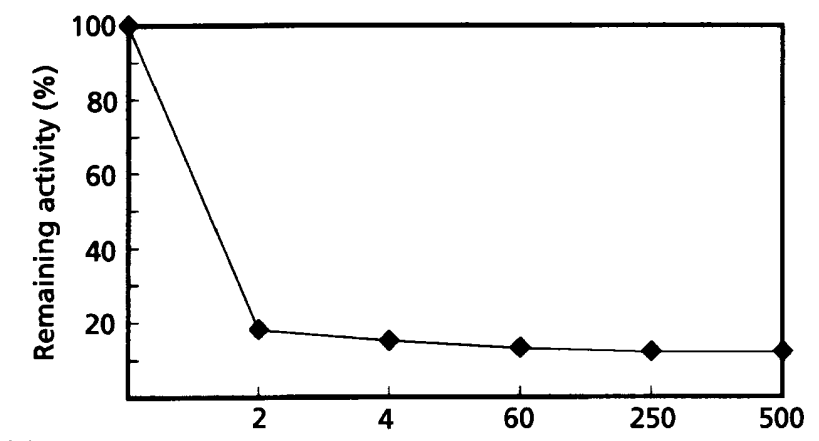

(b)

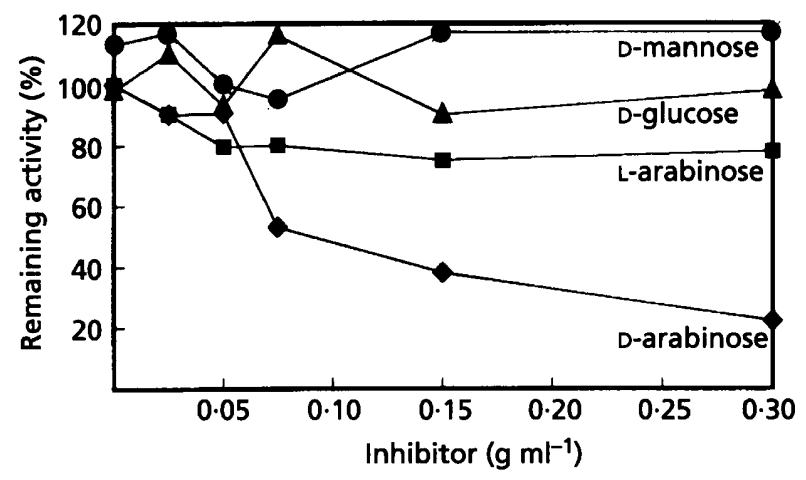

Fig. 6. Inhibition of the reaction between (a) a rabbit anti(whole lipid antigens of strain 51 of $M$. mucogenicum) serum and $25 \mu \mathrm{g}$ coated whole lipid extract of strain 51 by the LOS purified from the same strain and (b) a rabbit anti-(whole lipid antigens of strain $54 \mathrm{~s}$ of $M$. mucogenicum) serum and $25 \mu \mathrm{g}$ coated whole lipid extract of strain 545 by L-arabinose $(\square)$, Darabinose $(\diamond), D$-glucose $(A)$ and $D$-mannose $(O)$. One hundred per cent activity corresponded to an $A_{410}$ value of 1.77 .

strain 51 (Fig. 5). It was clearly demonstrated that this glycolipid was the main antigen of the lipid mixture by inhibition experiments in which up to $90 \%$ of the seroreactivity of the crude lipid from strain 51 with the homologous serum was inhibited by the LOS purified from the same strain (Fig. 6a).

\section{Chemical basis of the seroreactivity of the lipid extracts of isolate $54 \mathrm{~s}$}

The antigenicity of glycolipids is widely accepted as due to the carbohydrate moiety of the molecules. In mycobacteria, the presence of unusual sugar residues confers a structural diversity to the oligosaccharides that substitute a common lipid or lipopeptide core, which in turn governs the exquisite specificity of the seroreactivity of the surface-exposed glycolipids (see Brennan et al., 1990). Consequently, the $\mathrm{L}$ - and $\mathrm{D}$-isomers of arabinose, D-mannose and D-glucose, which composed the LOS of isolate $54 \mathrm{~s}$, were used to inhibit the reactivity of the lipid extract of strain $54 \mathrm{~s}$ with the serum directed against the lipid extract from the same strain (Fig. 6b). D-Arabinose inhibited up to $70 \%$ of the seroreactivity, whereas the Lisomer achieved only a $20 \%$ inhibition; as expected, neither glucose, which is the ubiquitous sugar constituent of all the mycobacterial LOS characterized so far (see Brennan et al., 1990), nor mannose, inhibit the reaction. These results demonstrated that the Darabinosyl residue of the LOS is involved in the antigenicity of the molecule.

\section{DISCUSSION}

The present study was undertaken to identify and chemically characterize the species-specific glycolipids of $M$. mucogenicum, the most recent species to be included in the $M$. fortuitum complex. Examination of the glycolipid content of three reference strains and 32 clinical and environmental isolates belonging to $M$. mucogenicum demonstrated that all the smooth strains of this species elaborate antigenic species-specific alkalilabile and phosphorus-free neutral glycolipids, i.e. LOS. This observation is similar to that noted by Belisle \& 
Brennan (1989) in the case of Mycobacterium kansasii. It has to be noted, however, that no correlation between the presence of LOS and the colony morphology of the strains has been observed in either $M$. tuberculosis (Lemassu et al., 1992) or the third biovariant complex of M. fortuitum (Lanéelle et al., 1996). The acylated triglucoside, the simplest form of LOS described so far, characterized in some strains of this latter heterogeneous group of organisms (Lanéelle et al., 1996), is structurally similar to the one found in some strains of $M$. fortuitum (Besra et al., 1992). These strains probably belong to the third biovariant complex, since only acylated trehaloses have been found in the type strain and in wellcharacterized strains of M. fortuitum (Ariza et al., 1994; Gautier et al., 1992; Sempere et al., 1993). Apart from these two exceptions, the presence of LOS has not been reported in the other species of the $M$. fortuitum complex, i.e. $M$. chelonae, $M$. abscessus and $M$. peregrinum, which elaborate antigenic GPLs as major glycolipids (López Marín et al., 1991, 1994; Tsang et al., 1984). For this reason, the presence of a characteristic LOS pattern in all the smooth strains of $\mathrm{M}$. mucogenicum investigated in the present study clearly differentiates $M$. mucogenicum from the other species that compose the $M$. fortuitum complex, and supports the recent proposal which considers $M$. mucogenicum to be a new species. Furthermore, the sera raised against the lipid extracts of the smooth strains of M. mucogenicum did not react with the lipid antigens of the representative strains of the other members of the $M$. fortuitum complex; this suggests that serological studies may help in the identification of strains belonging to the new species.

In the smooth strains of $M$. mucogenicum examined, three different LOS patterns were observed. The LOS present in $76 \%$ of the smooth strains examined was chemically characterized as a tetraacylated pentasaccharide; the LOS typifying the remaining strains of M. mucogenicum differ from the former LOS, and from the previously described LOS, by their sugar composition and their seroreactivity. It is worthy of note that the variability in the LOS patterns in mycobacterial strains belonging to the same species had previously been observed in Mycobacterium gordonae. Goslee et al. (1976) recognized seven distinct serotypes of $M$. gordonae, the dominant surface antigens of which have subsequently been characterized by Brennan et al. (1982) as LOS. The chemical explanation of such variability has recently been given by Besra et al. (1993) through the structural elucidation of LOS from two strains of $M$. gordonae. The authors showed that each strain contained a set of four major LOS, whose structures differed according to the strain examined. The purified LOS reacted strongly with the antiserum raised against the homologous strain and only weakly with the heterologous strain, demonstrating that $M$. gordonae is a serocomplex based on the structures of the LOS. Thus, like GPLs that typify the 31 serovars of the $M$. avium complex (see Brennan et al., 1990), LOS may be responsible for the occurrence of serovars in a given mycobacterial species. In the case of M. mucogenicum, the present study suggests the existence of at least three serovars which differ one from the other by the structure and seroreactivity of their LOS. In this connection, several studies, notably in the $M$. avium complex, have demonstrated the usefulness of determining these serovars in epidemiological studies (Falkinham, 1994; Kiehn et al., 1985; McClatchy, 1981; Shaefer, 1979; Yakrus \& Good, 1990). There is no doubt that epidemiological markers remain an important tool in the fight against pathogenic bacteria and fundamentally against those that give rise to epidemics. This is the case in $M$. mucogenicum which has caused several nosocomial epidemics and has been recovered in several occasions from tap water in the affected hospitals (Band et al., 1982; Bolan et al., 1985; Lowry et al., 1990). Interestingly, strains $54 \mathrm{~s}$ and 147 , which contain the same arabinose-containing LOS, were isolated from two patients who occupied the same ward in a hospital block for 2 weeks. Although neither of the two isolations were clinically significant, the two isolates possibly belong to the same clone that was contaminating the tap water of the hospital block at that time.

Finally, based on partial sequencing of the $16 \mathrm{~S}$ rRNA gene of the three reference strains of $M$. mucogenicum used in the present study, Springer et al. (1995) recently proposed the biochemically typical strain ATCC 49650 as the type strain of the new species rather than the previously recommended (Wallace et al., 1993) but atypical strain ATCC 49649. In our hands, however, the newly proposed type strain is atypical in terms of LOS content. This strain is the only one that elaborated galactose-containing LOS among the 32 isolates we examined. In conclusion, we recommend that strain ATCC 49651 be considered the type strain of $M$. mucogenicum, firstly because ATCC 49651 is a biochemically typical strain of $M$. mucogenicum, secondly because it contains the most often encountered LOS characterized in the present study, and thirdly because it has a $16 \mathrm{~S}$ rRNA gene sequence identical to that of the newly proposed type strain ATCC 49650 (Springer $e t$ al., 1995).

\section{ACKNOWLEDGEMENTS}

The authors are grateful to Mr L. Tran (Université Paul Sabatier, Toulouse, France) and J. D. Bounéry (Institut de Pharmacologie et de Biologie Structurale du CNRS, Toulouse, France) for their excellent technical assistance in recording the NMR and GC-MS spectra, respectively. We also thank Miss P. Féménia for her help in the first stage of this work. This work has been supported by grants from the Comision Interministerial de Ciencia y Tecnología PTR94-0080 and Fondo de Investigaciones Sanitarias de la Seguridad Social 96/1422.

\section{REFERENCES}

Ariza, M. A., Martín-Luengo, F. \& Valero-Guillén, P. L. (1994). A family of diacyltrehaloses isolated from Mycobacterium fortuitum. Microbiology 140, 1989-1994.

Band, J. D., Ward, J. I., Fraser, D. W., Peterson, N. J., Silcox, V. A., 
Good, R. C., Ostrey, P. R. \& Kennedy, J. (1982). Peritonitis due to a Mycobacterium chelonae-like organism associated with intermittent chronic peritoneal dialysis. $J$ Infect Dis 145, 9-17.

Belisle, J. T. \& Brennan, P. J. (1989). Chemical basis of rough and smooth variation in mycobacteria. J Bacteriol 171, 3465-3470.

Besra, G. S., McNeil, M. \& Brennan, P. J. (1992). Characterization of the specific antigenicity of Mycobacterium fortuitum. Biochemistry 31, 6504-6509.

Besra, G. S., McNeil, M. R., Khoo, K. H., Dell, A., Morris, H. R. \& Brennan, P. J. (1993). Trehalose-containing lipooligosaccharides of Mycobacterium gordonae: presence of a mono-O-methyltetra$\mathrm{O}$-acyltrehalose 'core' and branching in the oligosaccharide backbone. Biochemistry 32, 12705-12714.

Bolan, G., Reingold, A. L., Carson, L. A. \& 10 other authors (1985). Infections with Mycobacterium chelonae in patients receiving dialysis and using processed hemodialyzers. J Infect Dis 152, 1013-1019.

Bradbury, J. H. \& Jenkins, G. A. (1984). Determination of the structures of trisaccharides by ${ }^{13} \mathrm{C}-\mathrm{NMR}$ spectroscopy. Carbohydr Res 126, 125-156.

Brennan, P. J. \& Goren, M. B. (1979). Structural studies on the type-specific antigens and lipids of the Mycobacterium avium, Mycobacterium intracellulare and Mycobacterium scrofulaceum serocomplex. J Biol Chem 254, 4205-4211.

Brennan, P. J., Heifets, M. \& Ullom, B. P. (1982). Thin-layer chromatography of lipid antigens as a means of identifying nontuberculous mycobacteria. J Clin Microbiol 15, 447-455.

Brennan, P. J., Hunter, S. W., McNeil, M., Chatterjee, D. \& Daffe, M. (1990). Reappraisal of the chemistry of mycobacterial cell walls, with a view to understanding the roles of individual entities in disease processes. In Microbial Determinants of Virulence and Host Response, pp. 55-75. Edited by E. M. Ayoub, G. H. Cassell, W. C. Branche, Jr \& T. J. Henry. Washington, DC: American Society for Microbiology.

Daffé, M., Lacave, C., Lanéelle, M. A., Gillois, M. \& Lanéelle, G. (1988). Polyphthienoyl trehaloses, glycolipids specific for virulent strains of the tubercle bacillus. Eur J Biochem 172, 579-584.

Daffé, M., McNeil, M. \& Brennan, P. J. (1991). Novel type-specific lipooligosaccharides from Mycobacterium tuberculosis. Biochemistry 30, 378-388.

Falkinham, J. O., III (1994). Epidemiology of Mycobacterium avium infections in the pre- and post-HIV era. Res Microbiol 145 , 169-172.

Gautier, L., López Marín, L. M., Lanéelle, M. A. \& Daffé, M. (1992). Structure of mycoside F, a family of trehalose-containing glycolipids of Mycobacterium fortuitum. FEMS Lett 98, 81-88.

Goslee, S., Rynearson, T. K. \& Wolinsky, E. (1976). Additional serotypes of Mycobacterium scrofulaceum, Mycobacterium gordonae, Mycobacterium marinum, and Mycobacterium xenopi determined by agglutination. Int J Syst Bacteriol 26, 136-142.

Kiehn, T. E., Edwards, F. F., Brannen, P. \& 7 other authors (1985). Infections caused by Mycobacterium avium complex in immunocompromised patients: diagnosis by blood culture and fecal examination, antimicrobial susceptibility tests, and morphological and seroagglutination characteristics. J Clin Microbiol 21, 168-173.

Kirschner, P., Kiekenbeck, M., Meissner, D., Wolters, J. \& Bbttger, E. C. (1992). Genetic heterogeneity within Mycobacterium fortuitum complex species: genotypic criteria for identification. $J$ Clin Microbiol 30, 2772-2775.

Kusunoki, S. \& Ezaki, T. (1992). Proposal of Mycobacterium peregrinum sp. nov., nom. rev., and elevation of Mycobacterium chelonae subsp. abscessus (Kubica et al.) to species status: Mycobacterium abscessus comb. nov. Int J Syst Bacteriol 42, 240-245.

Lanéelle, M. A., Silve, G., López Marin, L. M. \& Daffé, M. (1996). Structures of the glycolipid antigens of members of the third biovariant complex of Mycobacterium fortuitum. Eur J Biochem 238, 270-279.

Lemassu, A., Lévy-Frébault, V. V., Lanéelle, M.-A. \& Daffé, M. (1992). Lack of correlation between colony morphology and lipooligosaccharide content in the Mycobacterium tuberculosis complex. J Gen Microbiol 138, 1535-1541.

Lévy-Frébault, V., Daffé, M., Goh, K. S., Lanéelle, M. A., Asselineau, C. \& David, H. L. (1983). Identification of Mycobacterium fortuitum and Mycobacterium chelonei. J Clin Microbiol 17, 744-752.

Lévy-Frébault, V., Grimont, F., Grimont, P. A. D. \& David, H. L. (1986). Deoxyribonucleic acid and relatedness study of the Mycobacterium fortuitum-Mycobacterium chelonae complex. Int J Syst Bacteriol 36, 458-460.

López Marín, L. M., Lanéelle, M. A., Promé, D., Daffé, M., Lanéelle, G. \& Promé, J. C. (1991). Glycopeptidolipids from Mycobacterium fortuitum: a variant in the structure of cmycoside. Biochemistry 30, 10536-10542.

López Marín, L. M., Gautier, N., Lanéelle, M.-A., Silve, G. \& Daffé, M. (1994). Structures of the glycopeptidolipid antigens of $\mathrm{Myco-}$ bacterium abscessus and Mycobacterium chelonae and possible chemical basis of the serological cross-reactions in the $\mathrm{Myco-}$ bacterium fortuitum complex. Microbiology 140, 1109-1118.

Lowry, P. W., Beck-Sague, C. M., Bland, L. A., Aguero, S. M., Arduino, M. J., Minuth, A. N., Murray, R. A., Swenson, J. M. \& Jarvis, W. R. (1990). Mycobacterium chelonae infection among patients receiving high-flux dialysis in a hemodialysis clinic in California. J Infect Dis 161, 85-90.

McClatchy, J. K. (1981). The seroagglutination test in the study of nontuberculous mycobacteria. Rev Infect Dis 3, 867-870.

McNeil, M., Chatterjee, D., Hunter, S. W. \& Brennan, P. J. (1989). Mycobacterial glycolipids : isolation, structures, antigenicity, and synthesis of neoantigens. Methods Enzymol 179, 215-242.

Muñoz, M., Julián, E., Garcia-Barceló, M., Ausina, V. \& Luquin, M. (1997). Easy differentiation of Mycobacterium mucogenicum from other species of the Mycobacterium fortuitum complex by thin-layer and gas chromatography of fatty esters and alcohols. J Chromatogr B Biomed Appl 689, 341-347.

Ryhage, R. \& Stenhagen, E. (1960). Mass spectrometric studies. IV. Esters of monomethyl-substituted long chain carboxylic acids. Ark Kemi 15, 291-304.

Sauton, B. (1912). Sur la nutrition minérale du bacille tuberculeux. C R Acad Sci Ser III Sci Vie 155, 860-863.

Sempere, M. A., Valero-Guillén, P. L., de Godos, A. \& MartínLuengo, F. (1993). A triacyltrehalose containing 2-methylbranched unsaturated fatty acyl groups isolated from Mycobacterium fortuitum. J Gen Microbiol 139, 585-590.

Shaefer, W. B. (1979). Serological identification of atypical mycobacteria. Methods Microbiol 13, 323-344.

Silcox, V. A., Good, R. C. \& Floyd, M. M. (1981). Identification of clinically significant Mycobacterium fortuitum complex isolates. $J$ Clin Microbiol 14, 686-691.

Springer, B., Bottger, E. C., Kirschner, P. \& Wallace, R. J., Jr (1995). Phylogeny of the Mycobacterium chelonae-like organism based on partial sequencing of the $16 \mathrm{~S}$ rRNA gene and proposal of Mycobacterium mucogenicum sp. nov. Int J Syst Bacteriol 45, 262-267. 
Sweeley, C. C., Bentley, R., Makita, M. \& Wells, W. W. (1963). Gas-liquid chromatography of trimethylsilyl derivatives of sugars and related substances. J Am Chem Soc 85, 2497-2507.

Tsang, A. Y., Barr, V. L., McClatchy, J. K., Goldberg, M., Drupa, I. \& Brennan, P. J. (1984). Antigenic relationships of the Mycobacterium fortuitum-Mycobacterium chelonae complex. Int J Syst Bacteriol 34, 35-44.

Wallace, R. J., Jr, (1994). Recent changes in taxonomy and disease manifestations of the rapidly growing mycobacteria. Eur J Clin Microbiol Infect Dis 13, 953-960.

Wallace, R. J., Jr, Swenson, J. M., Silcox, V. A., Good, R. C., Tschen, J. A. \& Seabury Stone, M. (1983). Spectrum of disease due to rapidly growing mycobacteria. Rev Infect Dis 5, 657-679.

Wallace, R. J., Jr, Brown, B. A., Silcox, V. A. \& 8 other authors (1991). Clinical disease, drug susceptibility, and biochemical patterns of the unnamed third biovariant complex of $\mathrm{Myco}$ bacterium fortuitum. J Infect Dis 163, 598-603.

Wallace, R. J., Jr, Silcox, V. A., Tsukamura, M., Brown, B. A., Kilburn, J. O., Butler, W. R. \& Onyi, G. (1993). Clinical significance, biochemical features, and susceptibility patterns of sporadic isolates of the Mycobacterium chelonae-like organism. J Clin Microbiol 31, 3231-3239.

Yakrus, M. A. \& Good, R. C. (1990). Geographic distribution, frequency, and specimen source of Mycobacterium avium complex serotypes isolated from patients with acquired immunodeficiency syndrome. J Clin Microbiol 28, 926-929.

Received 27 May 1997; revised 1 September 1997; accepted 12 September 1997. 